Martin Heine* and Arthur Bikbaev

\title{
Molecular dynamics of neuronal information transfer
}

https://doi.org/10.1515/nf-2017-A042

\begin{abstract}
A detailed analysis of synapses as connecting elements between neurons is of central importance to understand the brain's cognitive performance and its constraints. Nowadays, state-of-the-art optical methods make possible to localize individual molecules in a living cell. In particular, the dynamics of molecular composition can be evaluated in smallest neuronal compartments, such as pre- and postsynaptic membrane. The monitoring of the distribution of receptors, ion channels, and adhesion molecules over time revealed their continuous stochastic motion. This is surprising, since the synapses are considered as accumulation sites anchoring these molecules. The direct manipulation of the lateral dynamics of glutamate receptors, in combination with classical electrophysiological approaches, demonstrated that such molecular dynamics is necessary for the induction of synaptic plasticity and, in turn, is influenced by synaptic activity. Therefore, the molecular dynamics requires further studies in the context of the brain function in health and disease.
\end{abstract}

Keywords: Synapse, surface mobility, synaptic transmission, plasticity

\section{Introduction}

A fascinating feature of the brain is the ability to extract from an ever-changing flow of sensory stimuli an information, which is biologically relevant for a given organism. On one hand, the experience can be stored for future reference as a memory trace, which can later be modified by subsequent meaningful events, thus evolve over time. On the other hand, brain has to retain the ability to trigger quick immediate responses to environmental stimuli.

\footnotetext{
*Corresponding author: Martin Heine, Research Group "Molekular Physiology" at Leibniz Institute for Neurobiology Magdeburg, Brenneckestraße 6, 39118 Magdeburg, Germany; Otto-von-Guericke-Universität Magdeburg, Center for Behavioral Brain Sciences, 39120 Magdeburg, Germany, Mail: mheine@lin-magdeburg.de Arthur Bikbaev, Research Group “Molekular Physiology” at Leibniz Institute for Neurobiology Magdeburg, Brenneckestraße 6, 39118 Magdeburg, Germany, Mail: abikbaev@lin-magdeburg.de
}

Such a wide spectrum of responsiveness in various time scales is an attribute of neuronal networks, in which the neuronal excitability and synaptic contacts between individual neurons play a crucial role. Neurons, together with glial cells, are the building blocks of neuronal networks. Being embedded in the network, each neuron receives signals from many other neurons via synaptic inputs, which are located on the soma, but mostly on the extensively branching processes called dendrites. For instance, a pyramidal cell in the CA1 region of the rat hippocampus was found to have up to 30,000 glutamatergic (excitatory) and 1,700 GABAergic (inhibitory) synaptic contacts distributed along dendritic branches of total length approx. $12,000 \mu \mathrm{m}$ (Megias et al., 2001). Therefore, a meaningful integration of incoming stimuli represents a daunting challenge, with the strength of individual synapses and their localization being essential parameters. Inhibitory synapses are mainly located in proximal dendrites that are closer to the soma and axonal hillock, whereas excitatory synapses are distributed along both proximal and distal dendrites (Gulyas et al., 1999; Megias et al., 2001).

Dendritic structures can powerfully influence the integration of synaptic information via various mechanisms. The electric properties of dendritic membrane and different expression of voltage-dependent ion channels along the dendritic arbour can either amplify, or attenuate the somatodendritic representation of individual synapses (Tran-Van-Minh et al., 2015). However, the directed transfer of information in the neuronal networks is determined primarily by the strength and the frequency of synaptic activation. Short repetitive activation of a synapse can lead either to facilitation, or to depression of synaptic transmission that occur within a few milliseconds to seconds. Depending on the pattern of activity, this can result in long-term changes in the efficacy of synaptic transmission that can last for minutes and hours. The mechanisms that can change the properties of synaptic transmission in such a short time window are predominantly presynaptic and involve the accumulation of calcium in presynaptic terminal, reduction in ready releasable pool and retrograde signalling. However, postsynaptic mechanisms, such as desensitization or saturation of postsynaptic receptors, can also play a role (Zucker and Regehr, 2002). Rapid activity-dependent alterations at synaptic level 
are an important variable for storage of activity changes over time and the short-term memory (Abbott and Regehr, 2004). Synaptic changes that occur over longer time intervals are often dominated by postsynaptic mechanisms, but can also be mediated by persistent alteration in the presynaptic neurotransmitter release. The long-term functional changes are often accompanied by structural modification of synaptic contacts and considered as cellular correlate of learning and memory formation. Notably, the transition between short-term and persistent changes in the synaptic connectivity is gradual.

In early studies, it was observed that the direction of short-term plasticity, such as facilitation or depression, depends on the postsynaptic cell, which determines the temporal integration of synaptic activity. This was demonstrated first in crustacean motor neurons, where synapses originating from the same neural fibre show different short-term plasticity at different muscle fibres (Atwood and Bittner, 1971). Later, similar observations were made in primary neuronal cultures and in mammalian brain (Bao et al., 2010; Branco et al., 2008; Markram et al., 1998; Pouille and Scanziani, 2004), where terminals of one pyramidal cell contacting different populations of neurons showed distinct short-term plasticity that varied among postsynaptic targets. Furthermore, the localization, as well as the molecular composition, of the synapse are crucial for its function. These two parameters were considered for a long time as a very stable element of neuronal networks. However, the kinetic properties of ion channels and ionotropic receptors, the specificity of adhesion molecules and synaptic modulation by metabotropic receptors provide a plethora of potential mechanisms to explain the synaptic plasticity. The observations of persistent synaptic turnover (Attardo et al., 2015; Engert and Bonhoeffer, 1999) that depends upon the exchange (recycling) of membrane-associated receptors (Kerchner and Nicoll, 2008; Kittler and Moss, 2003) gave a decisive impulse to postulate the importance of the molecular dynamics as a variable influencing both synaptic plasticity and integrity of neuronal networks. First of all, manipulation with dynamic processes in the postsynaptic membrane of glutamatergic synapses revealed that local endo/exocytosis of AMPA receptors, as well as their lateral diffusion and distribution in the membrane, substantially influence the synaptic plasticity (Carroll et al., 1999; Heine et al., 2008; Luscher et al., 1999; Penn et al., 2017). In the following sections, the surface diffusion of synaptic signalling molecules and its recently shown key role in synaptic plasticity will be reviewed in detail.

\section{Methods of measuring the molecular mobility in the membrane}

The activity and distribution of single molecules can be evaluated using electrophysiological and optical methods. The knowledge of kinetic parameters is a prerequisite for electrophysiological approach, while imaging requires the specific tagging of molecules with optically detectable particles. The electrophysiologcal methods utilise the kynetic properties of ion channels and ionotropic receptors and thus are applicable to electrically active proteins, whereas optical methods can be applied to virtually any molecule.

Electrophysiologically, mobile receptor populations can be identified by means of pharmacological isolation. This method, known as functional tagging, was first employed for analysis of local exchange of NMDA receptors (NMDARs) in glutamatergic synapses (Tovar and Westbrook, 1999). During evoked or spontaneous activity, synaptic NMDARs can be irreversibly blocked by MK801. After washout of unbound MK801, the remaining NMDAR-mediated currents reflect the replacement of blocked receptors by naïve receptors from extrasynaptic locations. It was found that the recovery of NMDAR-mediated currents can be measured already within a few minutes after treatment. Ongoing parallel processes, such as endocytosis and exocytosis, are substantially slower and require more than an hour. Thus, the lateral exchange of membrane-integrated NMDARs is a dominant mechanism for the fast recovery of NMDA currents. However, the contribution of naïve receptors newly inserted into the membrane cannot be ruled out completely. Similar strategies have been develeoped for specific populations of AMPA and $\mathrm{GABA}_{\mathrm{A}}$ receptors (Thomas and Smart, 2006).

Optical methods enable the monitoring of both populations and single molecules. For optical tagging, molecules need to be equipped with fluorescent particles, which absorb light and emit a specific fluorescence. There is also a possibility to employ other imaging approcahes, e.g. using small gold particles that utilize the changes in the absorption or in the local refractive index (Celebrano et al., 2011; Lasne et al., 2006; Ritchie and Kusumi, 2003).

The fisrt proof of the mobility of synaptic receptors was obtained in FRAP (fluorescence recovery after photobleach) experiments. The time course of recovery of fluorescence intensity in the bleached region allows to directly estimate the mobile fraction of tagged molecules, and can be used for analysis of diffusion properties. For example, the local diffusion of acetylcholin receptors in the postsynaptic membrane of neuromuscular junction 
was monitored in clawed frog tadpoles using fluorescently tagged $\alpha$-bungarotoxin (Young and Poo, 1983). The development of genetically encoded $\mathrm{pH}$-sensitive fluorophores (e.g., pHluorin) made possible the direct visualization of surface molecules in the membrane. The $\mathrm{pH}$-sensitivity of the fluorophore allows to distinguish the receptors in the membrane from those located in vesicles, which substantially simplifies the specific analysis of receptors' mobility in the membrane. However, expression of transgenic fluorescently tagged receptors can potentially lead to their competition with endogenous receptor population. As a consequence, the tagged receptor-based dynamics in FRAP experiments often exceeds the actual mobility of endogenous receptors.

In contrast, the direct monitoring of single endogenous molecules is much more informative. The individual motion of molecules can give an idea about their dwell time and density distribution in the membrane. This becomes particularly relevant when the location of individual molecules is important for their function, which was shown for the vesicular release of neurotransmitter. Activation of several, in extreme case a single, calcium channels is sufficient to trigger the release of one vesicle in a close (20-50 nm) proximity (Eggermann et al., 2012). Given the spatial dimension of most synapses, with a diameter of active zone in the range of $300-500 \mathrm{~nm}$, molecular motions can be relevant in influencing the properties of synaptic transmission. The typical values of diffusion constants (0.005-0.5 $\left.\mu \mathrm{m}^{2} / \mathrm{s}\right)$ mean a potential displacement for approx. $10-100 \mathrm{~nm}$ in $10 \mathrm{~ms}$. The number of voltage-gated calcium channels (VGCCs), adhesion molecules and receptors for cortical synapses (e.g., hippocampal CA3-CA1 synapse) is between 10-150 molecules. The question is how the density of molecules restricts their mobility.

The visualization of local distribution and motion in membrane compartments like synapses requires the direct detection of single molecules over time. In this context, the emission properties of the fluorophore, size, the affinity and valence of binding to the ligand (e.g., primary antibodies, nanobodies), as well as the tagging density are crucial parameters. Beside correlation fluorescent microscopy, camera-based detection of single molecules over time (single particle tracking, SPT) is the most widely applied technique. The local fluorescence maximum (centroid) allows to define the location of the fluorophore with a precision of $10-40 \mathrm{~nm}$ (Figure 1A). Once the localisation is performed for single images, the positions of individual fluorophores can be connected over time (at frame rate) to create their trajectories (motion paths) (Figure 1B). The frame rate for representation of diffusive motion depends upon the number of emitted photons per time unit, which determines the localisation precision for individual fluorophores. In experiments with living cells, one should find a compromise between the strength of light illumination, phototoxicity for the cell and precision of measurements. Therefore, individual trajectories often provide only a rough estimate of the reality (Figure 1C). The following analysis of these trajectories involves calculation of the mean square displacement (MSD) and enables to characterise the type of motion (free diffusion, restricted diffusion, etc.), explored surface and the diffusion coefficients (Figure 1D, E). Depending upon the used fluorophore, molecules can be monitored for a few seconds (single fluorescent dyes) or for several minutes (quantum dots). Later, the changes in diffusive behaviour can be directly correlated with the position in the membrane (Figure 2). Individual molecular interactions are often very transient and, due to limited temporal resolution, cannot be observed directly. Further statistical analysis based on the displacement of a molecule between two time points allows to estimate, regardless of individual observation periods, the dwell time and surface density of molecules within small membrane compartments (Hoze et al., 2012; Renner et al., 2017).

\section{Cellular membrane as a dynamic compartment}

In conjunction with the insulating properties of cellular membrane, the specific distribution of ion channels is critical for electrical activity of neurons. Apart from that, the thermal energy stored in biological membranes causes the permanent Brownian molecular motion of both lipids and the membrane-embedded proteins. The maintenance of local differences in density of ion channels, for instance in the synaptic membrane or in axon hillock, depends on their anchoring and the existing diffusion barriers in the membrane. Therefore, the diffusive behaviour of individual molecules in the cellular membrane is very irregular and influenced by direct and indirect interactions with neighbouring molecules. Here, the lateral interactions within the membrane, as well as vertical interactions with membrane-associated structures on the inner and outer leaflet of the membrane, play a dominant role. The liberty of lateral mobility within cellular membrane is determined by the density of integral protein complexes and the local lipid composition of the membrane. An impressive example of inhomogeneous membrane organisation is the axonal initial segment (AIS). With its very high density of ion channels, AIS acts as initiation area of the action poten- 


\section{Analysis of the surface diffusion of membrane associated molecules}
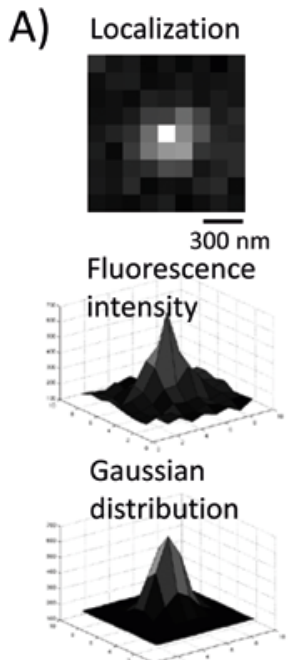

Subpixel localization

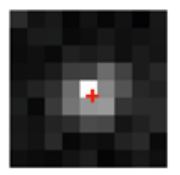

B)
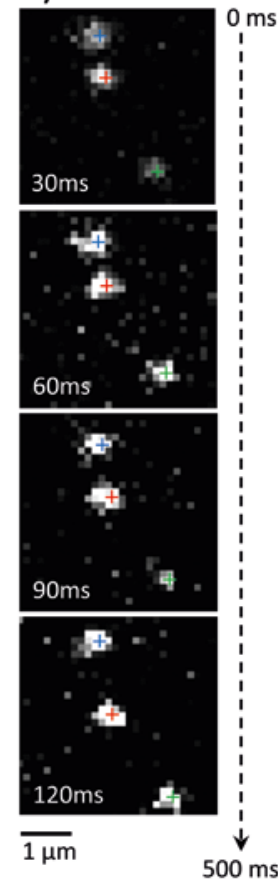

Localization of fluorophores $0 \mathrm{~ms}$ over time

$+$

+
+
+
+
++
+
++
++
++
++
++

$+$

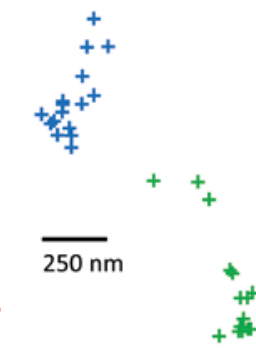

Trajectories of fluorophores in $\mathrm{X} / \mathrm{Y}$

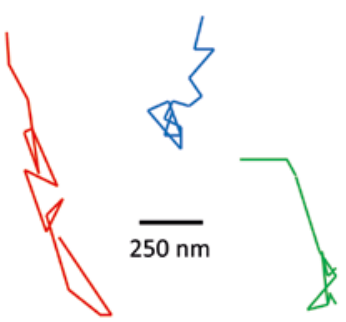

C) Simulation of higher acquisition rate
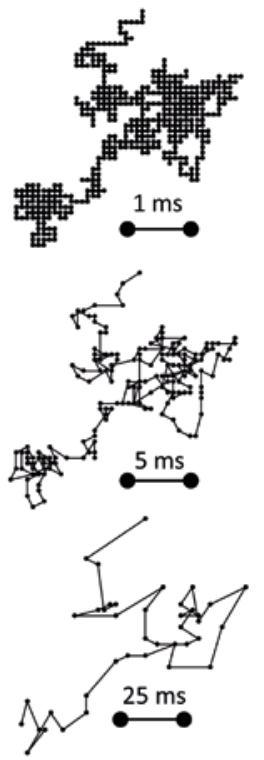

D)

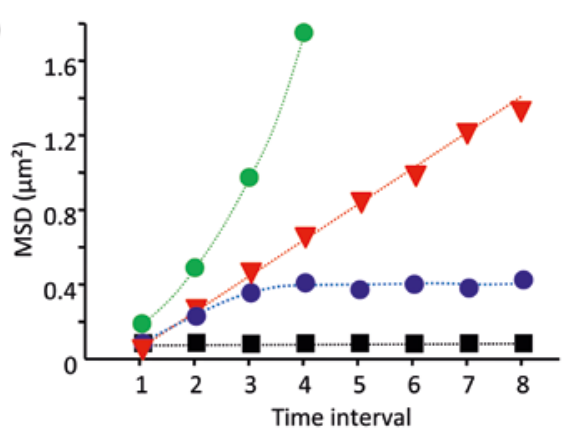

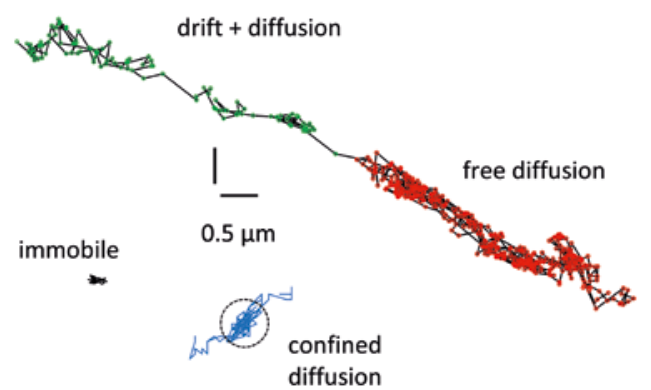

Fig. 1: Analysis of molecular mobility in the cellular membrane with Single Particle Tracking (SPT). A) Shown are the steps needed to localize the fluorophore in the plane of the picture. B) Time series for three fluorophores allow to create localisation maps, as well as trajectories of individual molecules over time. Those trajectories can be used for further analysis of the diffusive behaviour of the molecule and the explored surface. C) Simulation of a higher acquisition rate illustrates the improved representation of the explored surface over time. Following parameters were used: $1 \mathrm{~s}$ total acquisition time at acquisition rates of 25,100 , and 200 frames per second, the diffusion coefficient was set to $0.2 \mu \mathrm{m}^{2} / \mathrm{s}$. D) The quantification of diffusion coefficient and diffusion behaviour can be based on the calculation of the mean square displacement (MSD) over time interval. The deviation from free Brownian diffusion can be clearly visualised by the plot of the MSD versus time. Free Brownian diffusion (red), diffusion plus direct motion (green), confined diffusion (blue) and immobile particle (black) are depicted, with examples of trajectories along neurites represented in the corresponding colours.

tial, as well as diffusion barrier between somatodendritic and axonal compartments (Nakada et al., 2003; Winckler et al., 1999). Being present in all tubular processes of a neuron, cytoskeleton is built by actin rings that are bound via link protein adducin to spectrin filaments laid along the axon (Xu et al., 2013). In AIS, cytoskeleton serves as an anchor for scaffold proteins such as Ankyrin-G and thereby acts as an aggregation point for ion channels. In addition to the complexity of membrane composition, the tubular geometry of the membrane in neurites has impact on the diffusive motion of molecules (Domanov et al., 2011). The thinner neuronal processes are, the more restricted is the diffusion of membrane-associated molecules, which is particularly pronounced in thin post- 


\section{Single Particel Tracking in cultured brain slices}
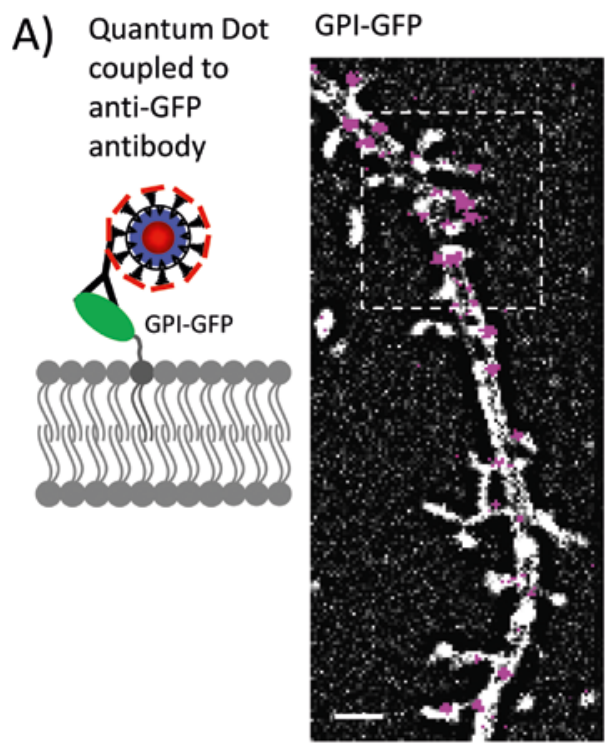

B)

Localisation of GPI-GFP molecules

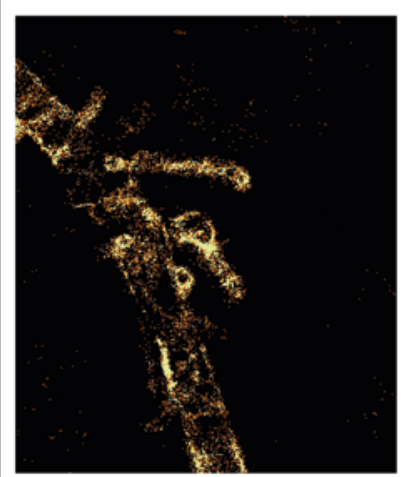

C)

$$
\begin{aligned}
& \text { Trajectories of individual } \\
& \text { GPI-GFP molecules }
\end{aligned}
$$

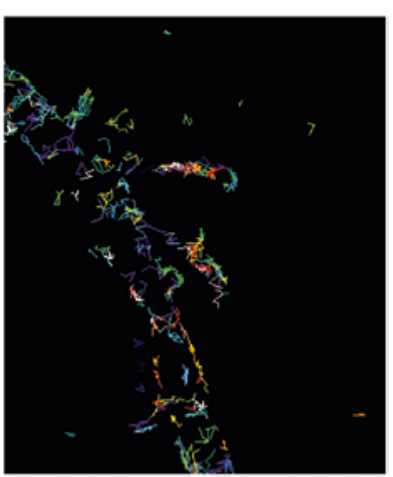

Fig. 2: Single Particle Tracking (SPT) in cultured brain slices. A) A schematic representation of surface molecule GPI-GFP, labelled with an anti-GFP antibody-coupled quantum dot (QD). QDs have several advantages that make them particularly interesting for SPT in cultured brain slices, namely their bright fluorescence, blinking emission and the broad excitation spectrum. These properties allow to detect QDs in the optically heterogeneous environment of a brain slice, to identify single QDs by their blinking emission and to reduce the impact of auto fluorescence in the preparation due to the large Stokes shift between excitation and emission of QDs. B) Position of single QDs (magenta) in the background image of a dendrite transfected with GPI-GFP. The tracking of individual QDs over time allows the reconstruction of the membrane outline through pooling the individual positions over time (here: 1000 frames within $30 \mathrm{~s}$ ). C) Reconstruction of trajectories is often complicated due to the blinking of the QDs and the crossover of neighbouring particles in the plane of view. For further information, see (Biermann et al., 2014).

synaptic spine necks. Further factors contributing to the non-uniform distribution of membrane proteins include local endo/exocytosis and formation of transmembrane protein clusters, interaction with extracellular matrix and electrostatic interactions (Trimble and Grinstein, 2015). The mobility of transmembrane proteins can be also influenced by the structure of the membrane itself and presence of membrane-associated structures on the surface.

\section{Dynamics of postsynaptic signal- ling proteins}

The dynamic distribution of receptors in the postsynaptic membrane was found equally important for the efficacy of information transfer in inhibitory and excitatory synapses. The mobility of receptors in glutamatergic synapses was studied most extensively, thus will be described further in detail.

Starting from the release of the glutamate into synaptic cleft, the diffusion of glutamate in extracellular space determines its signalling action as neurotransmitter. The release of one vesicle of glutamate is effective for signalling function within approx. $100 \mu$ s primarily due to low affinity of AMPA receptors (AMPARs) to glutamate. Depending on the composition, the $\mathrm{EC}_{50}$ value of AMPARs for glutamate is between $0.5-2.0 \mathrm{mM}$. This means that AMPARs within a radius of approx. $100 \mathrm{~nm}$ can be activated upon release of single vesicle, which contains 2,0005,000 glutamate molecules (Diamond and Jahr, 1997). In turn, the spatial arrangement, density and composition of postsynaptic AMPARs define the amplitude of postsynaptic current response. Since we focus here on the lateral mobility of molecules in the membrane, several questions may arise. How many receptors will be activated upon release of one vesicle with neurotransmitter? How stable is anchoring of receptors in the membrane? How the local density of receptors in the synapse can be modulated?

Synaptic structures seen in electron microscopic images suggest that stochastic motion of postsynaptic signalling proteins should be massively arrested due to their interconnections with membrane-associated scaffold proteins and the cytoskeleton. However, the intensive 
studies of the transport and the lateral diffusion of AMPARs revealed the fluctuation of receptors and associated signalling molecules as a central mechanism of synaptic plasticity. The analysis of lateral diffusion of receptors and lipids shows that diffusive behaviour of membrane-integrated molecules changes between free and restricted motion. For the receptors, such "stop and go" pattern potentially reflects their association/dissociation with intracellular scaffold proteins. AMPARs are accumulated in the synapse primarily through binding to PSD-95. In inhibitory GABAergic and glycinergic synapses, gephyrin plays similar role as central scaffold protein. Previously, the C-terminal binding motifs of individual AMPAR subunits to PSD-95 (C-terminal PDZ binding motif) were proposed as a basis of their direct interaction (Shi et al., 2001). However, these interactions of the receptor subunits with PSD-95 are too weak and could not fully explain stabilization of AMPARs upon induction of long-term synaptic plasticity (Kim et al., 2005). Instead, it appeared that synaptic anchoring of receptors can be mediated by other binding partners. Indeed, the discovery of receptor-associated proteins like TARPs (transmembrane AMPA receptor regulatory proteins), CKAMP44 (cysteine-knot AMPAR modulating protein 44), Shisa and Cornichon shed new light on the molecular organisation of postsynaptic membrane. These molecules are necessary for the intracellular transport of AMPARs to membrane and mediate their anchoring to scaffold proteins. Owing to the half-life of individual receptors or receptor-associated molecules, their stabilization in the synapse can last only for a short time. Thus, the transient interaction of receptors and associated proteins is an essential part of synaptic function and plasticity. Furthermore, the observation of individual receptors in the active synapse enables to better understand the temporal encoding of plasticity as a sequence of molecular interactions. In various studies of the function of TARPs, such flexible interactions were demonstrated in different time intervals.

Stargazin (TARPy-2), which was originally described as auxiliary y subunit of VGCCs (mice with stargazin mutation have characteristic stargazing posture), is important for the trafficking, synaptic surface expression and kinetic properties of AMPARs predominantly in the cerebellum. Moreover, TARPy-2 mediates the association of AMPARs with PSD-95 (Chen et al., 2000). The monitoring of individual receptors and TARPy-2 per se showed that the association of TARPy-2 with PSD-95 is essential for the stability and accumulation of AMPARs. This approach also demonstrated that the majority of receptors both within and outside the synapse is associated with TARPy-2 (Bats et al., 2007). Changes in synaptic activity can lead to phos- phorylation of C-terminus of TARPy-2, thereby affecting the binding affinity to PSD-95 (Opazo et al., 2010). Hence, the synaptic accumulation of AMPARs can be modulated within a time interval of seconds to a few minutes. The loss (mobilisation) or gain (immobilisation) of binding to intracellular scaffold proteins is in steady competition with available thermal energy of the membrane.

A particularly interesting scenario, which couples the association/dissociation between AMPARs and TARPy-2 to both the dynamics of AMPARs and their functional state, was recently proposed. Here, the association of receptor with TARPy-2 defines the sensitivity of the postsynaptic site to glutamate. Activation of the receptor upon binding to glutamate always leads to structural change in the extracellular domain of the receptor, which results in the opening of the channel. However, this glutamate-dependent conformational change is very short and followed by another change in conformation, which leads to desensitization of the receptor. In this state, AMPARs dissociate from TARPy-2 and lose the TARPy-2-mediated anchoring to scaffold protein PSD-95. The unbinding of glutamate sets the receptor back to closed but activatable state, and the receptor can again associate with TARPy-2.

It is conceivable that short-term loss of binding to PSD-95 can potentially be beneficial for the replacement of desensitized AMPARs by naïve receptors. The packing density of neighbouring integral proteins, also referred to as "molecular crowding", restricts the fast diffusion of desensitized receptors away from the synapse. Thus, the presynaptic release of glutamate initiates the displacement of postsynaptic receptors within a few nanometres (Constals et al., 2015).

These two examples show that indirect interactions of receptor with scaffold protein enable a substantially finer control of the receptor distribution. The proteomic analysis of AMPAR complexes revealed further binding partners that can be associated in different combinations with AMPARs (Schwenk et al., 2012; Schwenk et al., 2009). The non-uniform distribution of AMPAR subunits and associated proteins in different brain regions reflects a very complex molecular configuration of glutamatergic synapses, with the lateral mobility of AMPARs being functionally relevant for both short- and long-term synaptic changes.

\section{Surface diffusion of AMPARs as a variable of synaptic plasticity}

The exemplified mechanisms of dynamic redistribution of AMPARs lead to a question how stochastic changes in 
the receptor population can affect the synaptic plasticity. Postsynaptic variables of short-term plasticity include the kinetic properties of AMPARs that can be influenced by association with accessory proteins. In synapses with high release probability, a subpopulation of AMPARs is likely desensitized due to recent presynaptic stimulations and therefore contributes to the attenuation of the postsynaptic response. An artificial immobilisation of AMPARs during a repetitive synaptic activation leads towards even more pronounced synaptic depression (Heine et al., 2008). This demonstrates that lateral exchange of receptors, which implies the replacement of desensitized receptors by naïve ones that can be activated, partially compensates the depression (Figure 3A, B). The described above dissociation of receptors and TARPy-2 can explain how the lateral exchange of receptors can be driven by rapid changes in binding affinities to intracellular scaffold. Notably, association with other accessory proteins can result to different effects on the kinetic properties of AMPARs, particularly desensitisation (von Engelhardt et al., 2010). The dynamics of components of postsynaptic membrane should be therefore always considered together with the molecular composition, which varies strongly between different brain regions (Schwenk et al., 2014).

Long-term potentiation of synaptic transmission (LTP), postulated as one of the mechanisms of memory formation, can be triggered by changing the probability of vesicular release of neurotransmitter and/or by changes in the number, conductance and kinetic properties of receptors. The change in the number of AMPARs was described to be caused primarily by recruitment of receptors into the postsynaptic density. First, local exocytosis of intracellular AMPARs to postsynaptic membrane was proposed as a molecular mechanism. It is however plausible that a faster increase in the number of receptors can be achieved by recruitment of perisynaptic/extrasynaptic receptors that are already present in the membrane. Extrasynaptic AMPARs were functionally detected using iontophoretic or photolytic application of glutamate (Liu et al., 1999). Furthermore, tagging of individual receptors revealed that extrasynaptic population of AMPARs is very mobile (Heine et al., 2008; Liu et al., 1999). Using a combination of the direct manipulation of receptor recycling (endo/exocytosis) and restriction of lateral mobility by biotin-avidin-mediated cross-linking of receptors, the temporal order of receptors' recruitment to synapse has been demonstrated. Remarkably, persistent immobilisation of AMPARs in the cellular membrane leads to a complete blockade of LTP induction. The impairment of AMPAR exocytosis induced by perfusion of postsynaptic neurons with tetanus toxin has no effect on the transient post-tetanic potentiation, but completely prevents the induction of LTP (Figure 3C, D). Further variation of these two experiments in different experimental systems (in vivo, acute and cultured slices) confirms that lateral mobility of AMPARs is required for LTP induction. Moreover, restriction of lateral mobility of AMPARs in vivo can block the hippocampus-dependent fear conditioning at initial phase (Penn et al., 2017). Apparently, rapid redistribution of receptors is a necessary process to respond to acute changes in synaptic activity, and to maintain thereby a "mobile memory" of activity. The composition of receptor complex comprising various interaction partners can strongly affect such "mobile memory".

\section{Surface diffusion of presynaptic signalling proteins}

Using localisation microscopy methods, it was shown that presynaptic signalling molecules, such as VGCCs, SNARE proteins and adhesion molecules, diffuse in the membrane (Bademosi et al., 2017; Fu and Huang, 2010; Neupert et al., 2015; Schneider et al., 2015). However, little is known about the impact of such dynamics on the release of synaptic vesicles. Given the low density of VGCCs in the presynaptic membrane, one can speculate that a dynamic localisation is particularly relevant. As demonstrated in several studies, the vesicle and VGCC must be in a very close proximity $(<100 \mathrm{~nm})$ for the release of fusion-ready vesicle to occur (Eggermann et al., 2012). In conjunction with rather low affinity of vesicular calcium sensors to free intracellular calcium, short opening time of VGCCs defined by action potential's duration and the control of intracellular calcium by calcium-binding proteins, determine the spatial dimension (Eggermann et al., 2012). In this scenario, mobile VGCCs provide the possibility to temporarily reach the required proximity to the vesicle. The above mentioned influence of the post-synapse on the release probability points to different complexes of adhesion molecules that participate in the local organisation of presynaptic membrane (Missler et al., 2003; Sylwestrak and Ghosh, 2012). Another factor that tunes the probability of "transient coupling" between the VGCC and vesicle implies direct and indirect interaction of channels with scaffold proteins. Direct protein-protein interactions of the C-terminus of VGCC with Rab interacting molecule (RIM) and RIM binding protein have massive impact on the accumulation and positioning of VGCCs (Acuna et al., 2016; Kaeser et al., 2011). In turn, interaction of these proteins with other scaffold proteins in the cytomatrix of presyn- 


\section{Contribution of mobile AMPARs to short-term plasticity}

A) Mobile AMPA receptors

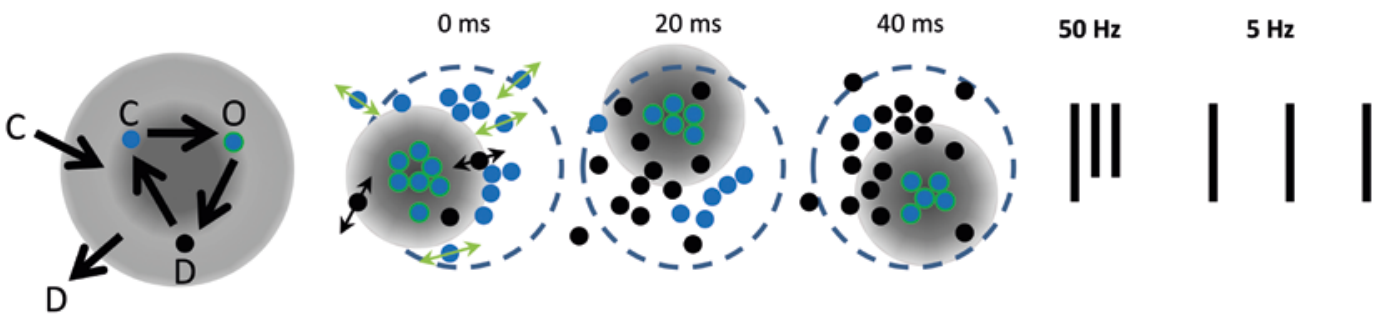

B) arteficially immobilized AMPA receptors
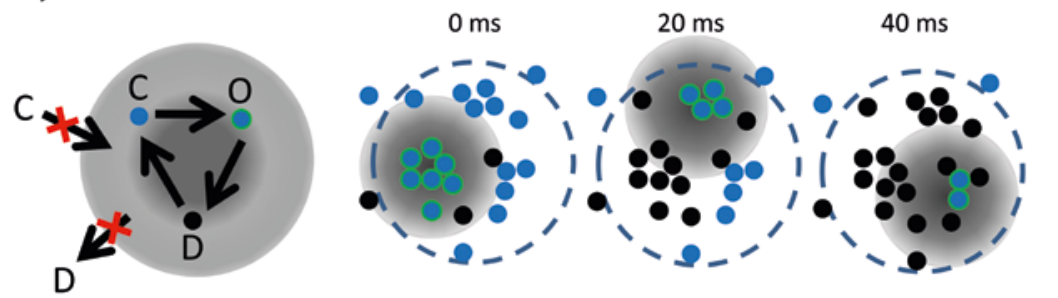

$50 \mathrm{~Hz}$

$5 \mathrm{~Hz}$

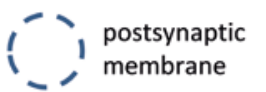

Glutamate release of one vesicle
open
AMPAR (O)
- closed
$\operatorname{AMPAR}(\mathrm{C})$

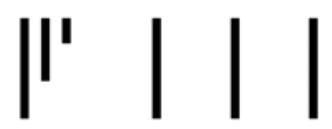

\section{Contribution of mobile AMPARs receptors to long-term potentiation}

C) postsynaptic block of AMPAR exocytosis

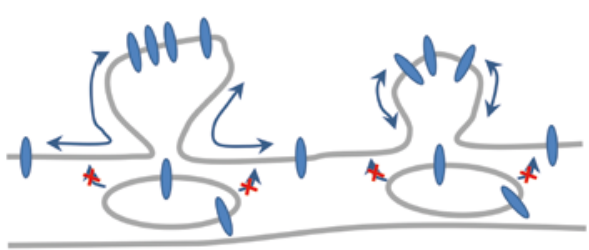

D) postsynaptic block of AMPAR surface mobility

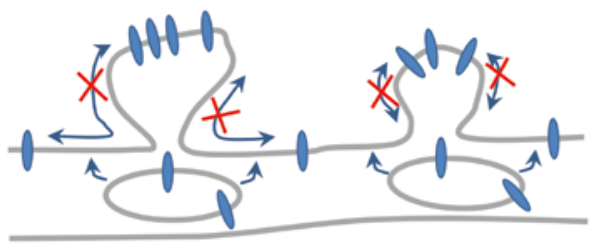

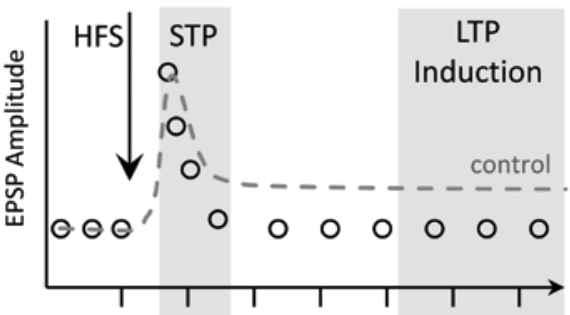

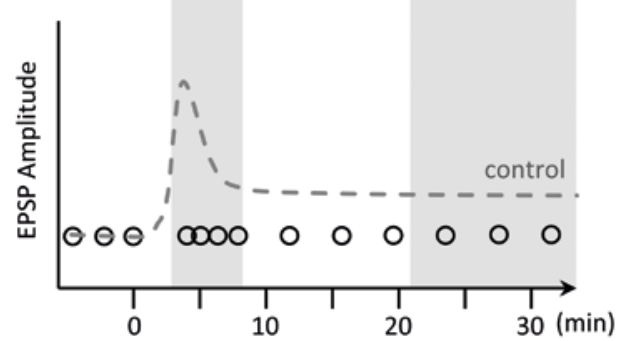

Fig. 3: Schematic representation of the impact of mobile AMPARs on synaptic activity and plasticity. A) The kinetic states of AMPAR that are relevant for the impact of AMPARs' diffusion on short-term plasticity. Here, the recovery from desensitisation is the time-limiting factor that underlies an increasing population of desensitized receptors over time in case of high-frequency synaptic activation ( $>10 \mathrm{~Hz})$. The individual indicated kinetic states are: closed receptors (C), open receptors (O) and desensitized receptors (D). In case of mobile AMPARs in the membrane, an accumulation of desensitized AMPARs can be partially compensated by lateral diffusion. B) An artificial cross-link of surface AMPARs induces a stronger depression of high-frequency stimulated synapses as indicated in the sketch, whereas responses to lower frequencies $\leq 5 \mathrm{~Hz}$ are not affected by AMPARs' immobilisation (see also Heine et al. 2008). C) Illustration of the contribution of AMPARs to the long-term synaptic potentiation. The postsynaptic blockade of AMPAR exocytosis by tetanus toxin prevents the induction of LTP (within first 30 min after tetanisation), whereas the post-tetanic potentiation or short-term potentiation (STP) remain unaltered. D) Global immobilisation of surface AMPARs before and after tetanisation completely prevents plastic changes of the synapse, modified after Penn et al. 2017. 
apse (e.g., Bassoon, Munc13, ELKS) are also involved into recruitment and positioning of channels (Biederer et al., 2017).

Estimation of the local density of pre- (RIM) and postsynaptic (PSD-95) scaffold proteins using localisation microscopy methods (STORM/PALM) supports the existence of so called nanocolumns, which optimise the interplay between the vesicle release and the density of postsynaptic receptors (Tang et al., 2016). Such nanocolumns can serve as both transmembranal obstacles and intracellular dock station for diffusive VGCC, thereby defining their activity range. On the other hand, the mobility of VGCCs (Schneider et al., 2015) makes possible that individual channels and ready releasable vesicles meet each other. The time span of such proximal interaction should have direct influence on the number of vesicles to be released. The monitoring of the vesicle fusion over time shows that localisation of release sites always changes between two time points, thus supporting the idea of transient coupling of the channel and vesicle (Maschi and Klyachko, 2017; Tang et al., 2016).

An additional factor that defines the transient character of coupling between channel and vesicle is the small number of VGCCs in the presynaptic membrane. At resting membrane potential, around $50 \%$ of VGCCs are inactivated, i.e. functionally unavailable, and cannot contribute to neurotransmitter release and synaptic transmission. In this context, a continues shuffling of calcium channels in the presynaptic membrane is likely to increase the chance of coupling between active channel and a vesicle and can, at least partially, compensate this effect.

\section{Overview}

Growing body of evidence accumulated in last decades shows an astonishing structural complexity of both preand postsynaptic molecular machinery in central synapses of mammalian brain. With the development of new imaging techniques and molecular tools, it becomes clearer that chemical synapse is not static, but a very dynamic system. The stochastic dynamics of membrane-integrated proteins is particularly meaningful for interactions of various proteins with very variable binding affinities. Thus, high flexibility is possible even in small spatial domains. The dynamic organisation of the synapse over time plays an important role in the formation and maturation of synapses, as well as for the induction of synaptic plasticity. In relation to recent studies of the function of mobile AMPARs, one can state that activity-triggered changes in surface dynamics of receptors and ion channels can be considered as a "molecular memory" of recent synaptic activity. Albeit, it is kept for only short period of time, spatial rearrangement of membrane-associated molecules provides a structural basis for storage of temporal features of previous input patterns. In any given moment, configuration of numerous molecular partners in crowded microenvironment of synapses powerfully affects the synaptic responses to the subsequent stimuli, thereby representing the initial stage of information processing and serving as a link from short-term modification of synaptic transmission to long-term memories in neuronal networks. Further investigation of molecular dynamics in synapses will help to better understand the plasticity of neuronal networks. Potentially, studies taking into account the brain region-specific expression of molecules can help in developing novel strategies to treat neuronal disorders. Recently, such an approach has been proposed for accessory AMPAR protein TARPy-8 (Maher et al., 2017).

Acknowledgment: We thank Dr. Ulrich Thomas, Jennifer Heck and Oliver Klatt for critics and corrections of the manuscript, as well as Dr. Andreas Voigt for Monte Carlo simulations of diffusive behaviour of single molecules in the membrane. We also thank the Federal State Sachsen-Anhalt (EFRE), the CBBS, the Leibniz Institute for Neurobiology (Magdeburg) and the Schram Foundation for financial support.

\section{Glossary}

\begin{tabular}{|c|c|}
\hline AMPAR & $\begin{array}{l}\alpha \text {-amino-3-hydroxy-5-methyl-4-isoxazole } \\
\text { propionic acid (AMPA) receptor }\end{array}$ \\
\hline Cornichon & $\begin{array}{l}\text { CNIHs are small, three-transmembrane-domain } \\
\text { proteins that bind directly to AMPAR }\end{array}$ \\
\hline CKAMP44 & cysteine-knot AMPAR modulating protein 44 \\
\hline ELKS & protein rich in the amino acids $E, L, K$ and $S$ \\
\hline GPI-GFP & $\begin{array}{l}\text { glycosylphosphatidylinositol linked to green } \\
\text { fluorescent protein, used as reporter of lipid } \\
\text { dynamics in the outer leaflet of the membrane }\end{array}$ \\
\hline LTP & Long-term potentiation \\
\hline Munc13 & priming factor for vesicular fusion \\
\hline NMDAR & $\mathrm{N}$-methyl-D-aspartate receptor \\
\hline PDZ & PSD-95/discs large/zonula occludens-1 \\
\hline PSD-95 & $\begin{array}{l}\text { postsynaptic density protein 95, which has } \\
\text { three PDZ-domains and a MAGUK-domain } \\
\text { (membrane-associated guanylate kinase) }\end{array}$ \\
\hline RIM & RAB interacting molecule \\
\hline $\begin{array}{l}\text { RIM binding protein } \\
\text { SNARE }\end{array}$ & $\begin{array}{l}\text { RAB interacting molecule binding protein } \\
\text { soluble } N \text {-ethylmaleimide-sensitive-factor } \\
\text { attachment receptor }\end{array}$ \\
\hline
\end{tabular}


Shisa

TARP

\section{References}

Abbott, L. F., and Regehr, W. G. (2004). Synaptic computation. Nature 431, 796-803.

Acuna, C., Liu, X., and Sudhof, T. C. (2016). How to Make an Active Zone: Unexpected Universal Functional Redundancy between RIMs and RIM-BPs. Neuron 91, 792-807.

Attardo, A., Fitzgerald, J. E., and Schnitzer, M. J. (2015). Impermanence of dendritic spines in live adult CA1 hippocampus. Nature 523, 592-596.

Atwood, H. L., and Bittner, G. D. (1971). Matching of excitatory and inhibitory inputs to crustacean muscle fibers. J Neurophysiol 34, 157-170.

Bademosi, A. T., Lauwers, E., Padmanabhan, P., Odierna, L., Chai, Y. J., Papadopulos, A., Goodhill, G. J., Verstreken, P., van Swinderen, B., and Meunier, F. A. (2017). In vivo single-molecule imaging of syntaxin $1 A$ reveals polyphosphoinositide- and activity-dependent trapping in presynaptic nanoclusters. Nat Commun 8, 13660.

Bao, J., Reim, K., and Sakaba, T. (2010). Target-dependent feedforward inhibition mediated by short-term synaptic plasticity in the cerebellum. J Neurosci 30, 8171-8179.

Bats, C., Groc, L., and Choquet, D. (2007). The interaction between Stargazin and PSD-95 regulates AMPA receptor surface trafficking. Neuron 53, 719-734.

Biederer, T., Kaeser, P. S., and Blanpied, T. A. (2017). Transcellular Nanoalignment of Synaptic Function. Neuron 96, 680-696.

Biermann, B., Sokoll, S., Klueva, J., Missler, M., Wiegert, J. S., Sibarita, J. B., and Heine, M. (2014). Imaging of molecular surface dynamics in brain slices using single-particle tracking. Nat Commun 5, 3024.

Branco, T., Staras, K., Darcy, K. J., and Goda, Y. (2008). Local dendritic activity sets release probability at hippocampal synapses. Neuron 59, 475-485.

Carroll, R. C., Beattie, E. C., Xia, H., Luscher, C., Altschuler, Y., Nicoll, R. A., Malenka, R. C., and von Zastrow, M. (1999). Dynamin-dependent endocytosis of ionotropic glutamate receptors. Proc Natl Acad Sci U S A 96, 14112-14117.

Celebrano, M., Kukura, P., Renn, A., and Sandoghdar, V. (2011). Single-molecule imaging by optical absorption. Nature Photonics 5, 95.

Chen, L., Chetkovich, D. M., Petralia, R. S., Sweeney, N. T., Kawasaki, Y., Wenthold, R. J., Bredt, D. S., and Nicoll, R. A. (2000). Stargazin regulates synaptic targeting of AMPA receptors by two distinct mechanisms. Nature 408, 936-943.

Constals, A., Penn, A. C., Compans, B., Toulme, E., Phillipat, A., Marais, S., Retailleau, N., Hafner, A. S., Coussen, F., Hosy, E., et al. (2015). Glutamate-Induced AMPA Receptor Desensitization
Increases Their Mobility and Modulates Short-Term Plasticity through Unbinding from Stargazin. Neuron 85, 787-803.

Diamond, J. S., and Jahr, C. E. (1997). Transporters buffer synaptically released glutamate on a submillisecond time scale. J Neurosci 17, 4672-4687.

Domanov, Y. A., Aimon, S., Toombes, G. E., Renner, M., Quemeneur, F., Triller, A., Turner, M. S., and Bassereau, P. (2011). Mobility in geometrically confined membranes. Proc Natl Acad Sci U S A 108, 12605-12610.

Eggermann, E., Bucurenciu, I., Goswami, S. P., and Jonas, P. (2012). Nanodomain coupling between $\mathrm{Ca}(2)(+)$ channels and sensors of exocytosis at fast mammalian synapses. Nat Rev Neurosci 13, 7-21.

Engert, F., and Bonhoeffer, T. (1999). Dendritic spine changes associated with hippocampal long-term synaptic plasticity. Nature 399, 66-70.

Fu, Y., and Huang, Z. J. (2010). Differential dynamics and activity-dependent regulation of alpha- and beta-neurexins at developing GABAergic synapses. Proc Natl Acad Sci U S A 107, 22699-22704.

Gulyas, A. I., Megias, M., Emri, Z., and Freund, T. F. (1999). Total number and ratio of excitatory and inhibitory synapses converging onto single interneurons of different types in the CA1 area of the rat hippocampus. J Neurosci 19, 10082-10097.

Heine, M., Groc, L., Frischknecht, R., Beique, J. C., Lounis, B., Rumbaugh, G., Huganir, R. L., Cognet, L., and Choquet, D. (2008). Surface mobility of postsynaptic AMPARs tunes synaptic transmission. Science 320, 201-205.

Hoze, N., Nair, D., Hosy, E., Sieben, C., Manley, S., Herrmann, A., Sibarita, J. B., Choquet, D., and Holcman, D. (2012). Heterogeneity of AMPA receptor trafficking and molecular interactions revealed by superresolution analysis of live cell imaging. Proc Natl Acad Sci U S A 109, 17052-17057.

Kaeser, P. S., Deng, L., Wang, Y., Dulubova, I., Liu, X., Rizo, J., and Sudhof, T. C. (2011). RIM proteins tether Ca2+ channels to presynaptic active zones via a direct PDZ-domain interaction. Cell 144, 282-295.

Kerchner, G. A., and Nicoll, R. A. (2008). Silent synapses and the emergence of a postsynaptic mechanism for LTP. Nat Rev Neurosci 9, 813-825.

Kim, C. H., Takamiya, K., Petralia, R. S., Sattler, R., Yu, S., Zhou, W., Kalb, R., Wenthold, R., Huganir, R., and M-334* (2005). Persistent hippocampal CA1 LTP in mice lacking the C-terminal PDZ ligand of GluR1. Nat Neurosci 8, 985-987.

Kittler, J. T., and Moss, S. J. (2003). Modulation of GABAA receptor activity by phosphorylation and receptor trafficking: implications for the efficacy of synaptic inhibition. Curr Opin Neurobiol 13, 341-347.

Lasne, D., Blab, G. A., Berciaud, S., Heine, M., Groc, L., Choquet, D., Cognet, L., and Lounis, B. (2006). Single nanoparticle photothermal tracking (SNaPT) of 5-nm gold beads in live cells. Biophys J 91, 4598-4604.

Liu, G., Choi, S., and Tsien, R. W. (1999). Variability of neurotransmitter concentration and nonsaturation of postsynaptic AMPA receptors at synapses in hippocampal cultures and slices. Neuron 22, 395-409.

Luscher, C., Xia, H., Beattie, E. C., Carroll, R. C., von Zastrow, M., Malenka, R. C., and Nicoll, R. A. (1999). Role of AMPA receptor cycling in synaptic transmission and plasticity. Neuron 24 , 649-658. 
Maher, M. P., Matta, J. A., Gu, S., Seierstad, M., and Bredt, D. S. (2017). Getting a Handle on Neuropharmacology by Targeting Receptor-Associated Proteins. Neuron 96, 989-1001.

Markram, H., Wang, Y., and Tsodyks, M. (1998). Differential signaling via the same axon of neocortical pyramidal neurons. Proc Natl Acad Sci U S A 95, 5323-5328.

Maschi, D., and Klyachko, V. A. (2017). Spatiotemporal Regulation of Synaptic Vesicle Fusion Sites in Central Synapses. Neuron 94, 65-73 e63.

Megias, M., Emri, Z., Freund, T. F., and Gulyas, A. I. (2001). Total number and distribution of inhibitory and excitatory synapses on hippocampal CA1 pyramidal cells. Neuroscience 102, 527-540.

Missler, M., Zhang, W., Rohlmann, A., Kattenstroth, G., Hammer, R. E., Gottmann, K., and Sudhof, T. C. (2003). Alpha-neurexins couple $\mathrm{Ca} 2+$ channels to synaptic vesicle exocytosis. Nature 423, 939-948.

Nakada, C., Ritchie, K., Oba, Y., Nakamura, M., Hotta, Y., lino, R., Kasai, R. S., Yamaguchi, K., Fujiwara, T., and Kusumi, A. (2003). Accumulation of anchored proteins forms membrane diffusion barriers during neuronal polarization. Nat Cell Biol 5, 626-632.

Neupert, C., Schneider, R., Klatt, O., Reissner, C., Repetto, D., Biermann, B., Niesmann, K., Missler, M., and Heine, M. (2015). Regulated Dynamic Trafficking of Neurexins Inside and Outside of Synaptic Terminals. J Neurosci 35, 13629-13647.

Opazo, P., Labrecque, S., Tigaret, C. M., Frouin, A., Wiseman, P. W., De Koninck, P., and Choquet, D. (2010). CaMKII triggers the diffusional trapping of surface AMPARs through phosphorylation of stargazin. Neuron 67, 239-252.

Penn, A. C., Zhang, C. L., Georges, F., Royer, L., Breillat, C., Hosy, E., Petersen, J. D., Humeau, Y., and Choquet, D. (2017). Hippocampal LTP and contextual learning require surface diffusion of AMPA receptors. Nature 549, 384-388.

Pouille, F., and Scanziani, M. (2004). Routing of spike series by dynamic circuits in the hippocampus. Nature 429, 717-723.

Renner, M., Wang, L., Levi, S., Hennekinne, L., and Triller, A. (2017). A Simple and Powerful Analysis of Lateral Subdiffusion Using Single Particle Tracking. Biophys I 113, 2452-2463.

Ritchie, K., and Kusumi, A. (2003). Single-particle tracking image microscopy. Methods Enzymol 360, 618-634.

Schneider, R., Hosy, E., Kohl, J., Klueva, J., Choquet, D., Thomas, U., Voigt, A., and Heine, M. (2015). Mobility of calcium channels in the presynaptic membrane. Neuron 86, 672-679.

Schwenk, J., Baehrens, D., Haupt, A., Bildl, W., Boudkkazi, S., Roeper, J., Fakler, B., and Schulte, U. (2014). Regional diversity and developmental dynamics of the AMPA-receptor proteome in the mammalian brain. Neuron 84, 41-54.

Schwenk, J., Harmel, N., Brechet, A., Zolles, G., Berkefeld, H., Muller, C. S., Bildl, W., Baehrens, D., Huber, B., Kulik, A., et al. (2012). High-resolution proteomics unravel architecture and molecular diversity of native AMPA receptor complexes. Neuron 74, 621-633.

Schwenk, J., Harmel, N., Zolles, G., Bildl, W., Kulik, A., Heimrich, B., Chisaka, O., Jonas, P., Schulte, U., Fakler, B., et al. (2009). Functional proteomics identify cornichon proteins as auxiliary subunits of AMPA receptors. Science 323, 1313-1319.

Shi, S., Hayashi, Y., Esteban, J. A., and Malinow, R. (2001). Subunitspecific rules governing AMPA receptor trafficking to synapses in hippocampal pyramidal neurons. Cell 105, 331-343.
Sylwestrak, E. L., and Ghosh, A. (2012). Elfn1 regulates targetspecific release probability at CA1-interneuron synapses. Science 338, 536-540.

Tang, A. H., Chen, H., Li, T. P., Metzbower, S. R., MacGillavry, H. D., and Blanpied, T. A. (2016). A trans-synaptic nanocolumn aligns neurotransmitter release to receptors. Nature 536, 210-214.

Thomas, P., and Smart, T. G. (2006). Receptor Dynamics at the Cell Surface Studied Using Functional Tagging. In The Dynamic Synapse: Molecular Methods in Ionotropic Receptor Biology, Kittler, J. T. and Moss, S. J., eds. (Boca Raton (FL)).

Tovar, K. R., and Westbrook, G. L. (1999). The incorporation of NMDA receptors with a distinct subunit composition at nascent hippocampal synapses in vitro. J Neurosci 19, 4180-4188.

Trimble, W. S., and Grinstein, S. (2015). Barriers to the free diffusion of proteins and lipids in the plasma membrane. J Cell Biol 208, 259-271.

von Engelhardt, J., Mack, V., Sprengel, R., Kavenstock, N., Li, K. W., Stern-Bach, Y., Smit, A. B., Seeburg, P. H., and Monyer, H. (2010). CKAMP44: a brain-specific protein attenuating short-term synaptic plasticity in the dentate gyrus. Science 327, 1518-1522.

Winckler, B., Forscher, P., Mellman, I., and M-253* (1999). A diffusion barrier maintains distribution of membrane proteins in polarized neurons. Nature 397, 698-701.

Xu, K., Zhong, G., and Zhuang, X. (2013). Actin, spectrin, and associated proteins form a periodic cytoskeletal structure in axons. Science 339, 452-456.

Young, S. H., and Poo, M. M. (1983). Rapid lateral diffusion of extrajunctional acetylcholine receptors in the developing muscle membrane of Xenopus tadpole. J Neurosci 3, 225-231.

Zucker, R. S., and Regehr, W. G. (2002). Short-term synaptic plasticity. Annu Rev Physiol 64, 355-405.

Article note: German version available at https://doi.org/10.1515/nf2017-0042

\section{Bionotes}

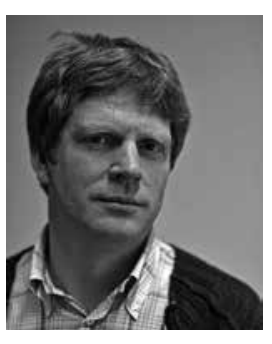

\section{Dr. Martin Heine}

Research Group “Molekular Physiology” at Leibniz Institute for Neurobiology Magdeburg, Brenneckestraße 6, 39118 Magdeburg, Germany; Otto-von-Guericke-Universität Magdeburg, Center for Behavioral Brain Sciences, 39120 Magdeburg, Germany

Mail: mheine@lin-magdeburg.de

Martin Heine studied biology at the Friedrich Schiller University Jena. In his PhD study he investigated the distribution of serotonin receptors in hippocampal neurons and their contribution to dendritic integration properties of CA1 neurons in the lab of Prof. D.W. Richter at the Georg August University in Göttingen. After the defence in 2002 Martin joined the group of Prof. D. Choquet in Bordeaux (France), where he investigated the functional contribution of AMPAR mobility to synaptic plasticity. In 2007 he went back to Germany and joined the Department of Neurochemistry 
at the Leibniz Institute of Neurobiology (LIN) headed by Prof. E. Gundelfinger. In 2009, M. Heine became an independent research group leader at the LIN. His main interests are the local dynamic organization of ion channels and adhesion molecules, as well as the impact of this stochastic variable on neuronal activity in neurons and neuronal networks.

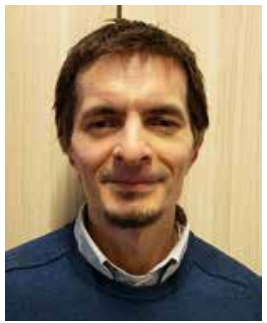

\section{Dr. Arthur Bikbaev}

Research Group "Molekular Physiology” at Leibniz Institute for Neurobiology Magdeburg, Brenneckestraße 6, 39118 Magdeburg, Germany Mail: abikbaev@lin-magdeburg.de

Arthur Bikbaev studied biology at Bashkir State University (Ufa, Russia). In his PhD study carried out at Bashkir State University and Lomonosov Moscow State University, he investigated the pathogenic mechanisms of the temporal lobe and absence forms of epilepsy. After defence, he moved to the group of Prof. D. Manahan-Vaughan at Ruhr University Bochum to study the relationship between hippocampal synaptic plasticity and network oscillations in vivo. In 2009, Arthur joined the group of Dr. Martin Heine to address the development of anatomic and effective connectivity in neuronal microcircuits in vitro and to analyse the transfer and processing of information in neuronal networks. 